\title{
Charge transport in a single molecule transistor probed by scanning tunneling microscopy $\dagger$
}

\author{
Samuel Bouvron, ${ }^{\mathrm{a}}$ Romain Maurand,$t^{\mathrm{b}}$ Alexander Graf, ${ }^{a}$ Philipp Erler, ${ }^{a}$ \\ Luca Gragnaniello, ${ }^{a}$ Maxim Skripnik, ${ }^{a}$ Dirk Wiedmann, ${ }^{a}$ Clara Engesser, ${ }^{a}$ \\ Cornelia Nef, ${ }^{\mathrm{b}}$ Wangyang Fu, ${ }^{\mathrm{b}}$ Christian Schönenberger, ${ }^{\mathrm{b}}$ Fabian Pauly ${ }^{\mathrm{a}}$ and \\ Mikhail Fonin (iD) ${ }^{a}$
}

\begin{abstract}
We report on the scanning tunneling microscopy/spectroscopy (STM/STS) study of cobalt phthalocyanine (CoPc) molecules deposited onto a back-gated graphene device. We observe a clear gate voltage $\left(V_{\mathrm{g}}\right)$ dependence of the energy position of the features originating from the molecular states. Based on the analysis of the energy shifts of the molecular features upon tuning $V_{\mathrm{g}}$, we are able to determine the nature of the electronic states that lead to a gapped differential conductance. Our measurements show that capacitive couplings of comparable strengths exist between the CoPc molecule and the STM tip as well as between CoPc and graphene, thus facilitating electronic transport involving only unoccupied molecular states for both tunneling bias polarities. These findings provide novel information on the interaction between graphene and organic molecules and are of importance for further studies, which envisage the realization of single molecule transistors with non-metallic electrodes.
\end{abstract}

\section{Introduction}

The perspective to exploit functional molecules in electronic or spintronic devices has generated considerable research activities focused on the investigation of electronic transport phenomena at the scale of single molecules. ${ }^{1-4}$ Charge transport at single molecule scale is usually studied by using metalmolecule-metal junctions, with the electromigration ${ }^{5}$ and the break junction ${ }^{6,7}$ techniques being the most popular ones. An important feature of these methods is the possibility of realizing a three-terminal device geometry in order to tune the molecular states that are relevant for the charge transport by using a back-gate electrode. ${ }^{8-12}$ However, in order to gain a profound control of the junction geometry in the molecular level transport experiments, the implementation of techniques is desirable, which are capable of atomic resolution, such as scanning tunneling microscopy (STM). Indeed, the possibility of incorporating a gate electrode within the STM geometry can favor-

${ }^{a}$ Department of Physics, University of Konstanz, D-78457 Konstanz, Germany. E-mail: samuel.bouvron@uni-konstanz.de,mikhail.fonin@uni-konstanz.de

${ }^{b}$ Department of Physics, University of Basel, Klingelbergstrasse 56, CH-4056 Basel, Switzerland

$\dagger$ Electronic supplementary information (ESI) available: The details of the theoretical modeling of the electronic transport; the results for the calculations based on the density functional theory including the influence of the electric field on the molecular orbital energies. See DOI: 10.1039/C7NR06860C \$Current address: CEA-Grenoble, LaTEQS, 38054 Grenoble Cedex 9, France. ably complement its high lateral resolution, giving an additional possibility of tuning the electronic and magnetic properties of nanoobjects accessible by STM. ${ }^{13-19}$ Recently, a number of experiments have been reported showing that the electronic properties of atoms ${ }^{20,21}$ or molecules, ${ }^{22}$ adsorbed onto graphene gated devices, can be tuned via application of a back-gate voltage $V_{\mathrm{g}}$, as observed by scanning tunneling spectroscopy (STS).

Here, we report on the impact of the gate voltage on the electronic levels of a single cobalt phthalocyanine (CoPc) molecule deposited on back-gated graphene/ $/ \mathrm{SiO}_{2} / \mathrm{Si}$ [see Fig. 1(a)]. The molecular features observed in differential conductance measurements show a rich and complex behavior under the application of a gate voltage. We analyze the energy shifts of the main features in a broad range of applied bias and gate voltages in order to determine the nature of the electronic states that lead to a gapped differential conductance in the STS spectra. We show that the observed behavior can be well explained assuming electronic transport involving only unoccupied molecular states.

\section{Methods}

Experimental setup

The experiments were carried out in an ultra-high vacuum (UHV) system ( $p=4 \times 10^{-11} \mathrm{mbar}$ ) equipped with an Omicron cryogenic STM. Electrochemically etched and in situ flash- 
(a)
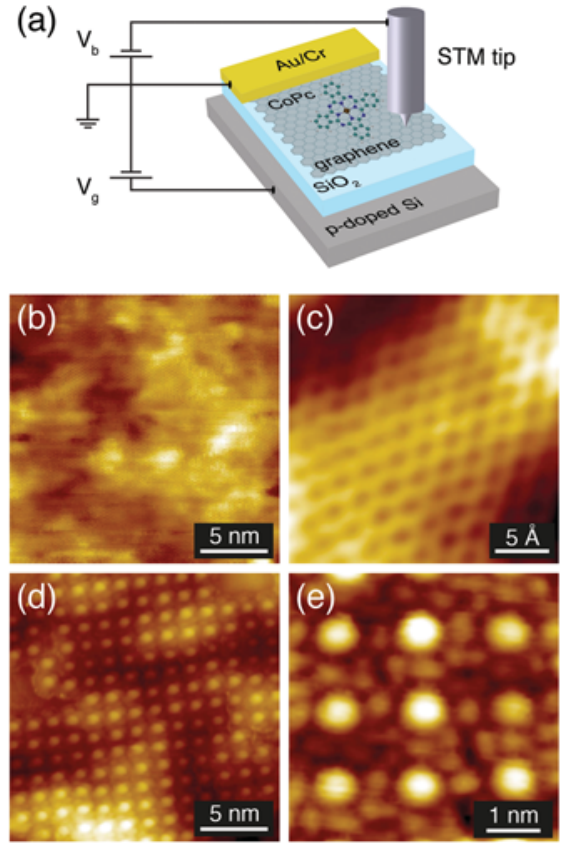

Fig. 1 (a) Schematic of the gated graphene device with CoPc molecules on the surface. (b) STM topographic image of graphene on $\mathrm{SiO}_{2} / \mathrm{Si}$ (image size: $30 \times 20 \mathrm{~nm}^{2}$, bias voltage: $V_{\mathrm{b}}=-0.3 \mathrm{~V}$, tunneling current: $I=1.5 \mathrm{nA}$, temperature: $T=10 \mathrm{~K}$ ). (c) Atomically resolved image of the graphene lattice $\left(2.5 \times 2.5 \mathrm{~nm}^{2}, V_{\mathrm{b}}=-0.4 \mathrm{~V}, I=0.4 \mathrm{nA}, T=12 \mathrm{~K}\right)$. (d) STM topography of a CoPc molecular layer on graphene $/ \mathrm{SiO}_{2} / \mathrm{Si}(30 \times$ $20 \mathrm{~nm}^{2}, V_{\mathrm{b}}=2 \mathrm{~V}, I=50 \mathrm{pA}, T=4 \mathrm{~K}$ ). (e) A part of the CoPc assembly with the intramolecular contrast $\left(5 \times 5 \mathrm{~nm}^{2}, V_{\mathrm{b}}=2.6 \mathrm{~V}, I=30 \mathrm{pA}, T=\right.$ $4 \mathrm{~K})$.

annealed tungsten tips were used for the STM measurements. The sign of the bias voltage $V_{\mathrm{b}}$ corresponds to the potential applied to the sample. $\mathrm{d} I / \mathrm{d} V_{\mathrm{b}}$ spectra and maps were recorded by means of a standard lock-in technique with a modulation voltage of $20-30 \mathrm{mV}$ (root-mean-square, rms) and a modulation frequency of $667.14 \mathrm{~Hz}$. The gate voltage $V_{\mathrm{g}}$ was applied using a Keithley electrometer 6517A, measuring the gate current continuously within all experiments to rule out any leakage $(<100 \mathrm{pA})$ or break down of the oxide layer.

\section{Sample preparation}

Monolayer graphene was prepared by low pressure chemical vapor deposition on a $25 \mu \mathrm{m}$ thick copper $(\mathrm{Cu})$ foil in a split quartz tube furnace. The transfer of graphene from the $\mathrm{Cu}$ foil onto a $\mathrm{SiO}_{2} / \mathrm{Si}$ substrate was performed by spin-coating of a $400 \mathrm{~nm}$ thick polymethylmethacrylate (PMMA) layer over graphene and by the subsequent etching of the $\mathrm{Cu}$ film in an ammonium persulfate solution. After deposition of the graphene/PMMA samples on the $\mathrm{SiO}_{2} / \mathrm{Si}$ substrates, yielding PMMA/graphene/ $/ \mathrm{SiO}_{2} / \mathrm{Si}$ stacks, PMMA was removed using acetone and a hydrogen annealing cycle at $700 \mathrm{~K}$. Graphene was electrically brought into contact by direct evaporation with gold (100 nm thick)/chromium (10 nm thick) electrodes through a shadow mask. Prior to the deposition of CoPc molecules, the samples were outgassed in the UHV at $700 \mathrm{~K}$.

\section{Theoretical modelling}

Different methods are applied in the theoretical modeling. DFT, as implemented in TURBOMOLE [http://www.turbomole. com], is used to compute molecular orbital wavefunctions of $\mathrm{CoPc}$ and their energies. They are then compared with the STM and STS images acquired at different bias voltages. In addition, in order to understand the behavior of the molecular transistor as a function of bias and gate voltages, a constant interaction model is used. It is complemented by a more sophisticated description that includes the electrostatic charging energy of the graphene layer.

\section{Results and discussion}

Prior to the deposition of CoPc molecules we investigate the surface quality of the graphene $/ \mathrm{SiO}_{2} / \mathrm{Si}$ device, whose geometry is depicted in Fig. 1(a). A topographic STM image of the pristine graphene is presented in Fig. 1(b) which reveals a clean surface with a typical vertical corrugation of $\sim 10 \AA$ (rms) imposed by the $\mathrm{SiO}_{2}$ surface roughness. ${ }^{23}$ The surface cleanness is clearly demonstrated by the atomically resolved STM image, showing a honeycomb graphene lattice [Fig. 1(c)]. After the deposition of CoPc molecules on graphene $/ \mathrm{SiO}_{2} / \mathrm{Si}$, large well-ordered islands of $\mathrm{CoPc}$ with an almost perfect quadratic unit cell are visible [Fig. 1(d and e)], as reported previously. ${ }^{24,25}$ The appearance of the CoPc molecules depends strongly on the bias voltage $\left(V_{\mathrm{b}}\right)$ as shown in Fig. $2(\mathrm{a}-\mathrm{d})$. The characteristic cross-like shape with the intensity spread over the center and four lobes is usually imaged at negative bias voltages [Fig. 2(a)]. For positive bias voltages the central protrusion dominates the contrast [Fig. 2(c)], which is usually attributed to the large contribution of the cobalt $(\mathrm{Co}) \mathrm{d}_{z^{2}}$ state to the tunneling transport. ${ }^{26}$ The accompanying density functional theory (DFT) calculations performed for the gas phase [Fig. 2(e)] confirm that the lowest unoccupied molecular orbital (LUMO) of the CoPc molecules is centered at the Co ion, while the highest occupied molecular orbital (HOMO) and second lowest unoccupied molecular orbital (LUMO+1) are spread over the lobes (see ESI S2 $\uparrow$ for further details of the DFT calculations).

Fig. 2(f) shows typical STS spectra obtained at the center and the lobes of a CoPc molecule. The spectra recorded at the center reveal a prominent peak at about $+1.1 \mathrm{~V}$ accompanied by satellite features appearing up to $+1.7 \mathrm{~V}$ and a small feature at about $+0.5 \mathrm{~V}$ [region P1 in Fig. 2(f)]. We attribute the main feature to the tunneling transport through the Co $\mathrm{d}_{z^{2}}$ orbital (LUMO) in accordance with the topographic measurements [Fig. 2(c)]. The observed satellites may arise from the contribution of excited states to the electronic transport ${ }^{8}$ or from vibronic transitions. ${ }^{27}$ The latter may become visible due to a weak coupling of CoPc molecules to the graphene substrate. ${ }^{22}$ Both the topographic images and $\mathrm{d} I / \mathrm{d} V_{\mathrm{b}}$ maps [Fig. 2(c) and (d)] show a pronounced intensity variation of the central region of $\mathrm{CoPc}$, when going from one molecule to another, indicating that the LUMO appears at a slightly different energy 

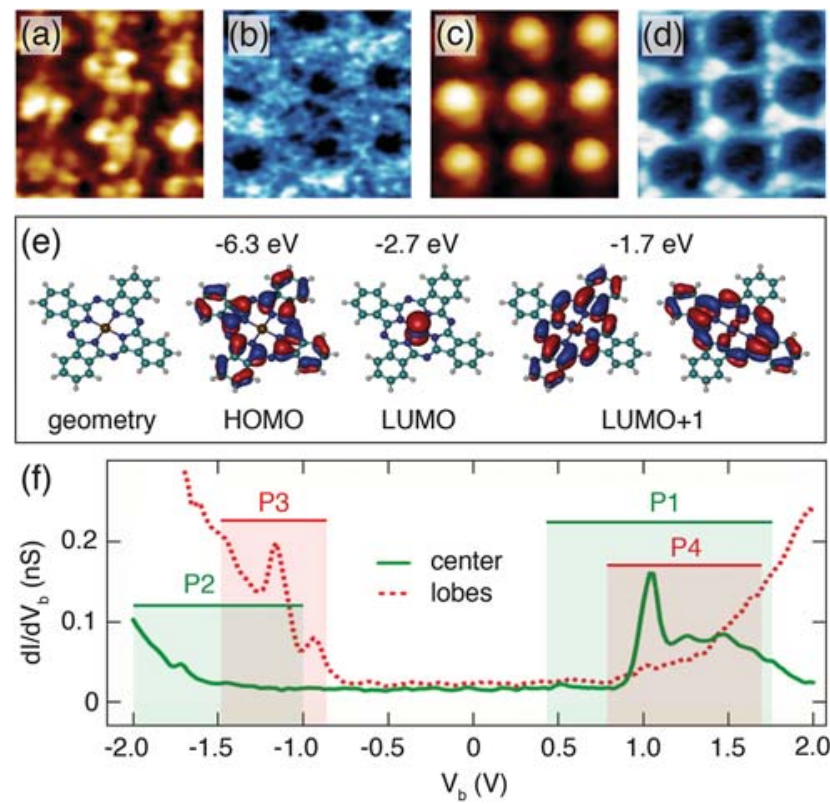

Fig. 2 (a) A $4 \times 4 \mathrm{~nm}^{2}$ topographic STM image of the CoPc layer and (b) a simultaneously recorded $\mathrm{d} l / \mathrm{d} V_{\mathrm{b}}$ map at $V_{\mathrm{b}}=-0.9 \mathrm{~V}\left(V_{\mathrm{g}}=0 \mathrm{~V} ; l=17\right.$ $\mathrm{pA} ; T=4 \mathrm{~K}$ ). (c) Topographic image of the same area and (d) a simultaneously obtained $\mathrm{d} l / \mathrm{d} V_{\mathrm{b}}$ map at $V_{\mathrm{b}}=2 \mathrm{~V}\left(V_{\mathrm{g}}=0 \mathrm{~V} ; I=50 \mathrm{pA} ; T=4 \mathrm{~K}\right)$. (e) Ground state geometry of gas-phase CoPc as determined using DFT, and the Kohn-Sham frontier molecular orbitals with their respective energies. The LUMO+1 state consists of two orbitals with the same energy but different spatial orientation. (f) Site-resolved STS spectra recorded at the center and at the lobes of a CoPc molecule. Set parameters: $V_{\mathrm{b}}=2 \mathrm{~V} ; V_{\mathrm{g}}=0 \mathrm{~V} ; I=100 \mathrm{pA} ; T=9 \mathrm{~K}$.

for each molecule. This observation suggests a variation of the interaction strength between graphene and CoPc molecules, which is mediated by the Co orbitals. ${ }^{28}$ Except for P1 features described above, only relatively flat signals in the range between -1.0 and $-2.0 \mathrm{~V}$ (region P2) are observed. These features will be further discussed in conjunction with the gatedependent measurements.

For the STS spectra taken at the lobes of CoPc molecules, pronounced peaks are only present in the negative bias voltage range $-1.5 \mathrm{~V} \leq V_{\mathrm{b}} \leq-0.8 \mathrm{~V}$ [region P3 in Fig. 2(f)]. The topographic STM image taken at $V_{\mathrm{b}}=-0.9 \mathrm{~V}$ [Fig. 2(a)] shows the characteristic cross-like shape of the molecule. The $\mathrm{d} I / \mathrm{d} V_{\mathrm{b}}$ map, recorded concomitantly to the topographic image, reveals a diffuse contribution originating from the lobes [Fig. 2(b)]. In accordance with the spectra presented in Fig. 2(f), no pronounced contribution from the Co $d_{z^{2}}$ state is observed. Additionally, a slope starting at about $+1.5 \mathrm{~V}$ [region P4 in Fig. 2(f)] appears. This contribution is further revealed in the $\mathrm{d} I / \mathrm{d} V_{\mathrm{b}}$ map at $+2 \mathrm{~V}$ [Fig. 2(d)], where only the outer ligands of the molecules are visible.

Fig. 3 shows the influence of the gate voltage $V_{g}$ on the energy positions of the features in the $\mathrm{d} I / \mathrm{d} V_{\mathrm{b}}$ spectra. For fixed values of $V_{\mathrm{g}}$, spectra were recorded both at the center [Fig. 3(a) and (c)] and at the lobes [Fig. 3(b) and (d)] of CoPc molecules. Scanning was continued between the acquisition of the different spectra to rule out spatial drift effects, i.e. the feedback was closed in between. Upon the application of a gate voltage $V_{\mathrm{g}}$ the features observed in the $\mathrm{d} I / \mathrm{d} V_{\mathrm{b}}$ spectra at $V_{\mathrm{g}}=0$ shift in energy, whereas the shift direction and the shift velocity (slopes in the $V_{\mathrm{g}} / V_{\mathrm{b}}$ diagram) are different for different features [Fig. 3(e) and (f)]. Upon increasing $V_{g}$ the peaks at $V_{\mathrm{b}}<0$ (P2 and P3) shift upwards in energy, whereas the features at $V_{\mathrm{b}}>0(\mathrm{P} 1$ and $\mathrm{P} 4)$ shift in the opposite direction, i.e. downwards in energy. Thus the features observed in the positive and negative $V_{\mathrm{b}}$ ranges cannot be simply attributed to different states, e.g. the HOMO and LUMO, because those would move in the same direction upon variation of the gate voltage. The change in the amplitude of the spectroscopic features can be explained in terms of variation of the tip-sample distance upon application of the gate voltage resulting from a varying number of molecular states contributing to the tunneling process. Because of the exponential dependence of the tunneling current on the distance, this is expected to have a negligible effect on the capacitive coupling discussed later.

In the following, we suggest that the tunneling transport involves mainly the unoccupied molecular states, where P1 and $\mathrm{P} 2$ are assigned to the LUMO, and P3 and P4 to the LUMO+1. Indeed, features shifting in opposite directions depending on the bias polarities are characteristic for single molecule transistors as well as the contribution to charge transport of a single molecular level for both bias polarities (bipolar transport). Such a behavior has already been observed for molecules deposited on a decoupling layer studied by $\mathrm{STM}^{29,30}$ and can be understood in terms of the variation of the electric potential in the tunneling junction, when the bias voltage is varied, with the capacitive coupling between the tip and molecule not being small compared to that between the molecule and sample.

To clarify this, we first consider the situation, when the electronic coupling between the STM tip and the cobaltphthalocyanine ( $\mathrm{CoPc}$ ) molecule is weak compared with that between graphene and CoPc. Two peaks appear in the differential conductance resulting from the transport through the HOMO and LUMO states at different bias voltages [see Fig. 4 $(\mathrm{a}-\mathrm{d})]$. If the gate potential is increased $\left(V_{\mathrm{g}}>0\right)$, the HOMO and LUMO states are lowered in energy as indicated by the red arrows. In the STS spectrum both peaks should shift in the same direction (as indicated by the red arrows) for both $V_{\mathrm{b}}$ polarities. However, this transport regime is obviously not compatible with our experimental observations.

Instead we consider a different type of transport regime, which can be realized in the case, when the tip is closer to the molecule, and the molecule's electronic coupling to the tip is comparable to its coupling to graphene. In this case, the local gating produced by the tip leads to the shift of a molecular state (occupied or unoccupied) upwards or downwards with respect to $E_{\mathrm{F}}$ depending on the bias polarity. As a consequence this state can solely contribute to transport for both bias polarities leading to the corresponding peaks in the $\mathrm{d} I / \mathrm{d} V_{\mathrm{b}}$ spectra. This situation is depicted in Fig. 4(e-h) for a LUMO state. As can be inferred from the red arrows, an increase of $V_{\mathrm{g}}$ makes the LUMO state shift closer to the $E_{\mathrm{F}}$ in energy and thus 

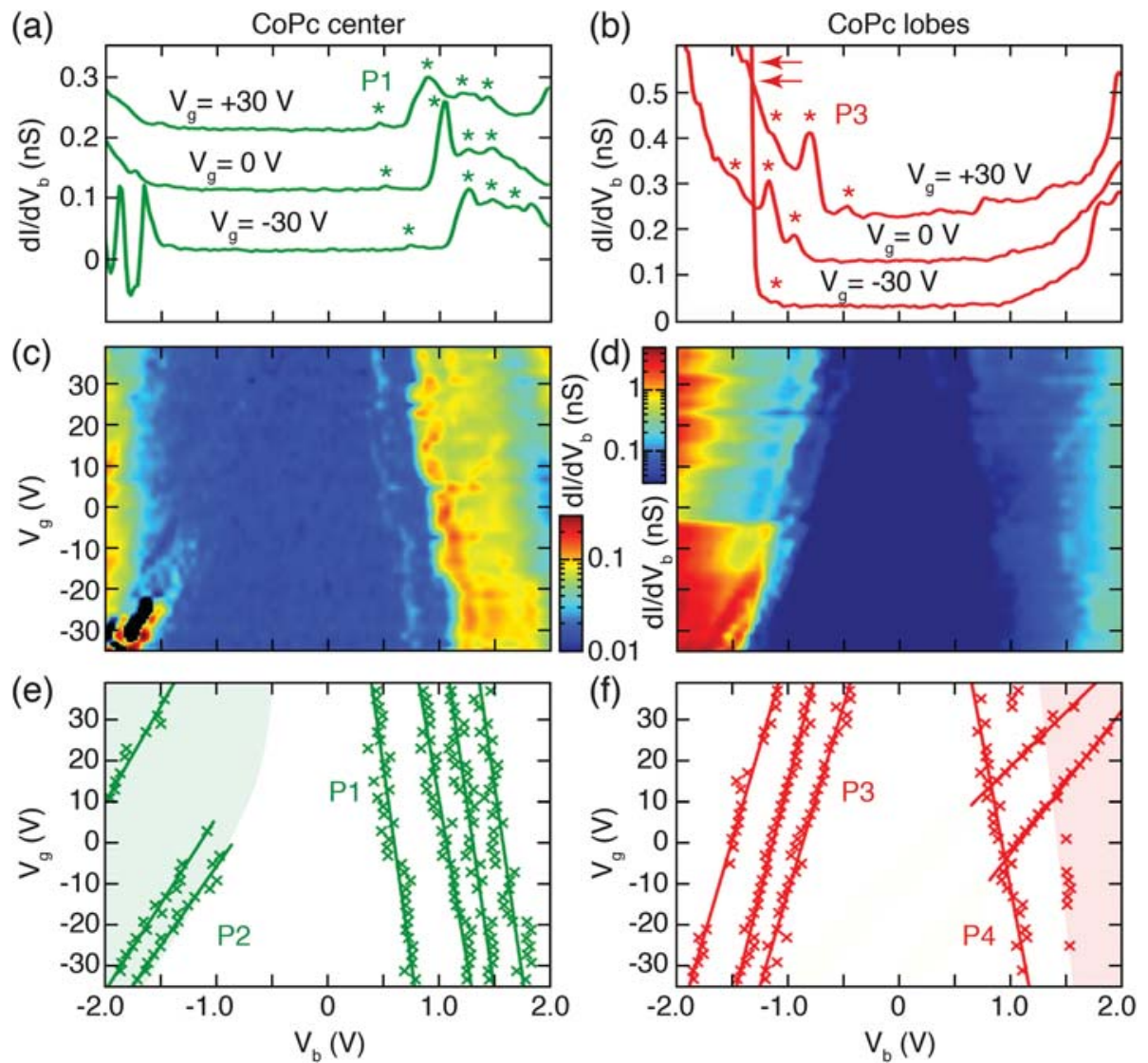

Fig. $3(a, b)$ Examples of $d / / d V_{b}$ spectra recorded at the center and at the lobes of a CoPc molecule for three different gate voltages. (c, d) Density plots of $\mathrm{d} / / \mathrm{d} V_{\mathrm{b}}$ spectra obtained from the spectra recorded for different gate voltages. Set parameters: $V_{\mathrm{b}}=2 \mathrm{~V} ; I=100 \mathrm{pA} ; T=9 \mathrm{~K}$. (e, f) Evolution of peak positions as a function of the applied gate voltage, as determined by the Lorentzian fit of the relevant features. Crosses mark the positions of the Lorentzian maxima for the features, which could be reliably fitted. Lines represent linear fits of the experimental data. Shaded areas indicate regions with a gate voltage induced contrast variation, where the peak positions could not be unambiguously determined.

reduces the $V_{\mathrm{b}}$ needed to make it contribute to transport. Consequently the apparent gap in the differential conductance decreases. In the simulated STS spectrum both peaks stemming from the LUMO move closer together. The opposite behavior would occur for a HOMO state: an increase of the gate potential leads to the increase of $V_{\mathrm{b}}$ at which a peak is observed in the $\mathrm{d} I / \mathrm{d} V_{\mathrm{b}}$ spectra, with the two peaks moving further apart, as can be seen in Fig. 3. Consequently, features related to the LUMO-mediated transport at negative bias move upwards in energy with increasing $V_{g}$, as observed in Fig. 3 for the features P2 and P3. This behavior must be due to the fact that the capacitive couplings between the STM tip and the $\mathrm{CoPc}$ molecule as well as between graphene and the CoPc molecule are comparable in strength. Thus, we assign the series of peaks P1 and P2 to the LUMO states, since those are obtained at the center of the CoPc molecule, and the series of peaks $\mathrm{P} 3$ and $\mathrm{P} 4$ to the LUMO+1 states, since those are recorded at the lobes. Because the tip is in a very close vicinity to the substrate, a gating of the graphene layer is expected upon application of a potential to the tip.

Fig. 5(a) shows the shift velocity $\mathrm{d} V_{\mathrm{g}} / \mathrm{d} V_{\mathrm{b}}$ as a function of the feature position at $V_{\mathrm{g}}=0$, thus summarizing the influence of the gate voltage on the features observed at the center and at the lobes of five different CoPc molecules. Only those features are shown, where the shift of the maximum could be unambiguously identified from the experiment. The small absolute values of $\mathrm{d} V_{\mathrm{g}} / \mathrm{d} V_{\mathrm{b}}$ correspond to a large influence of $V_{\mathrm{g}}$ on the energy position of the molecular states. The influence of $V_{g}$ also depends on the original energy position of the peaks at $V_{\mathrm{g}}=$ 0 . The larger the bias voltage needed to make a molecular state contribute to transport, the larger is the doping induced by the tip in the graphene layer, and the lower is the influence of the gate voltage. To better understand the experimental observations, we have used two theoretical models, sketched in Fig. 5. They both consider the transport and electrostatic coupling between the molecule as well as source (s), drain (d) and gate $(\mathrm{g})$ electrodes. As we will discuss now, they describe the system at different levels of sophistication, and both confirm that the gapped conductance observed in the experiment can be interpreted in terms of LUMO-mediated transport.

The first model (model 1) is based on the standard description of the Coulomb blockade in the constant interaction model, ${ }^{4,31}$ with the molecule representing a quantum dot which is coupled capacitively to the source (s), drain (d), and 
(a)

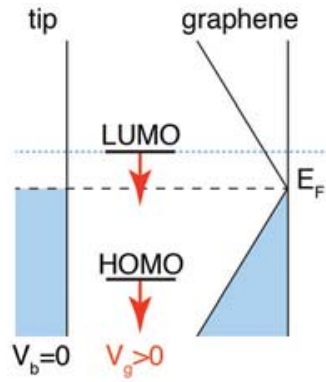

(e)

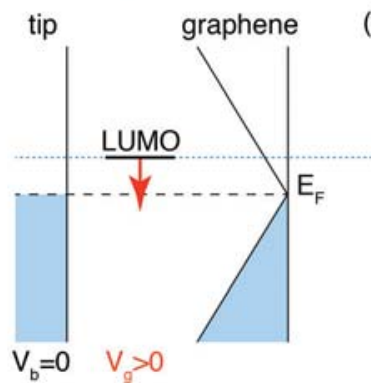

(b)

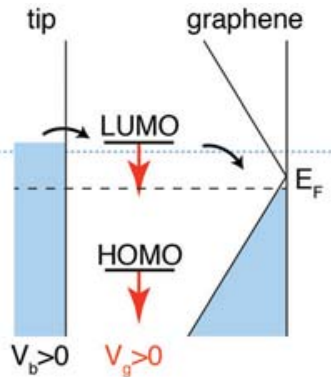

(f) tip (c)

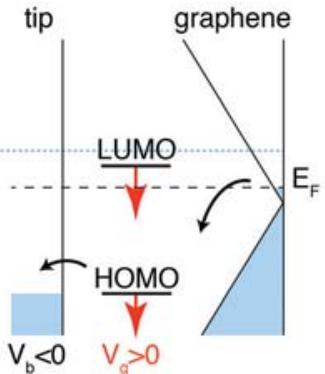

(d)

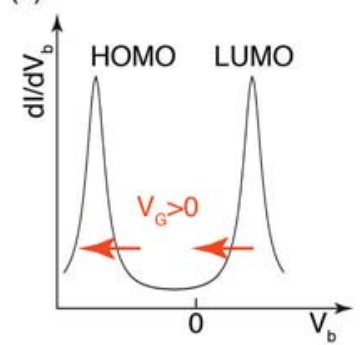

(h)

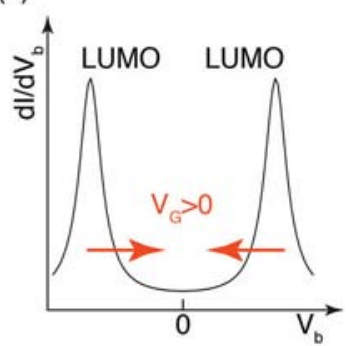

Fig. $4(a-d)$ The electronic coupling between the STM tip and the CoPc molecule is weak compared with the coupling between graphene and CoPc. Two peaks appear in the differential conductance resulting from a transport through the HOMO and LUMO states at different bias voltages. As indicated by the red arrows both peaks move in the same direction upon application of $V_{g}$. (e-h) The coupling between the tip and the CoPc molecule is comparable to the coupling between graphene and the CoPc molecule. The application of a positive gate potential reduces the apparent gap in the differential conductance in the case of the LUMO-mediated transport. The red arrows indicate the shift of the levels upon increasing of $V_{\mathrm{g}}$. In both scenarios, a deviation from charge neutrality in graphene due to the applied $V_{\mathrm{b}}$ is considered in addition to the effect of $V_{\mathrm{g}}$.
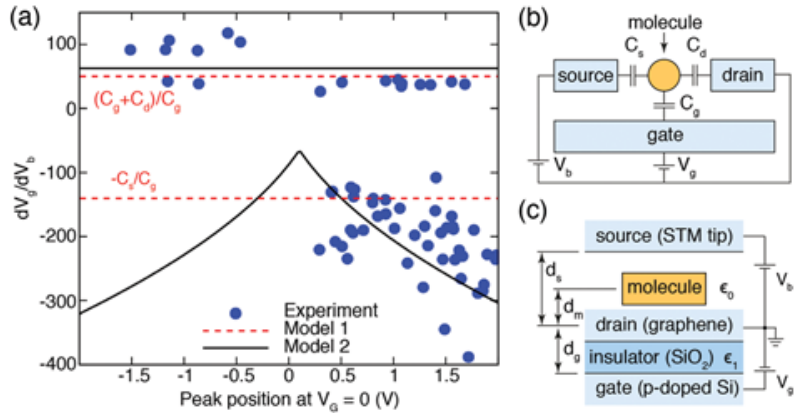

Fig. 5 (a) $d V_{\mathrm{g}} / \mathrm{d} V_{\mathrm{b}}$ as a function of the position of the peaks at $V_{\mathrm{g}}=$ 0 . Data points correspond to the slopes of the line fits to the experimental data as presented in Fig. 3. Results for two different theoretical models are shown in addition (red dashed and black solid lines). The first model (b), the constant interaction model, is based on the capacitive coupling of the molecular quantum dots to the electrodes. In the plot, we assumed $C_{d}=50 C_{g}$ and $C_{s}=140 C_{g}$. The second model (c) does not take the Coulomb blockade on the molecule into account. Instead, it describes transport in a phase-coherent single-particle picture and considers the electrostatic contributions between the source, drain, and gate electrodes as well as the charging energy of the graphene to determine the alignment of the molecular orbitals. The calculations were performed with $d_{\mathrm{s}}=1 \mathrm{~nm}, d_{\mathrm{m}}=0.2 \mathrm{~nm}, d_{\mathrm{g}}=300 \mathrm{~nm}, \varepsilon_{1}=4 \varepsilon_{0}, \Phi_{\mathrm{g}}=4.5 \mathrm{eV}$ and the Fermi velocity of graphene was set to $2 \times 10^{6} \mathrm{~m} \mathrm{~s}^{-1}$.

gate (g) electrodes via $C_{\mathrm{s}}, C_{\mathrm{d}}$ and $C_{\mathrm{g}}$, respectively. As shown in Fig. 5, tunneling is allowed between the source electrode and the quantum dot (the STM tip and the molecule) as well as between the quantum dot and the drain electrode (the molecule and the graphene). While the drain is grounded, the source is connected to a power supply with a voltage $V_{\mathrm{b}}$. Another power supply is used to adjust the electrochemical potential of the gate (the $\mathrm{Si}$ underneath the graphene/ $/ \mathrm{SiO}_{2}$ layer) through the gate voltage $V_{g}$, which enables the external control of the energy shift of the molecular orbitals. An enhanced current, induced by $V_{\mathrm{b}}$, flows through the molecule, if molecular orbitals enter the energy window, where electrochemical potentials of the source and drain differ. The alignment with the source's electrochemical potential leads to the relation $V_{\mathrm{g}}=\frac{1}{\beta} V_{\mathrm{b}}+V_{\mathrm{c}}$, and the alignment with that of the drain leads to $V_{\mathrm{g}}=-\frac{1}{\gamma} V_{\mathrm{b}}+V_{\mathrm{c}}$, where $\beta=C_{\mathrm{g}} /\left(C_{\mathrm{g}}+C_{\mathrm{d}}\right), \gamma=$ $C_{\mathrm{g}} / C_{\mathrm{s}}, V_{\mathrm{c}}=(N-1 / 2) e / C_{\mathrm{g}}+\left(C_{\mathrm{s}}+C_{\mathrm{g}}+C_{\mathrm{d}}\right) E_{\mathrm{N}} /\left(e C_{\mathrm{g}}\right)$ and $e=|e|$ is the absolute value of the elementary charge. The energy $E_{n}$ is the single-particle energy of the $n$-th electron in the molecule, measured relative to the equilibrium electrochemical potential of the source and drain electrodes. In a $V_{\mathrm{b}}-V_{\mathrm{g}}$ plot, the two relations define the areas of conducting behavior, ${ }^{4,31}$ as shown in Fig. 5(a). Their intersection at $V_{\mathrm{b}}=0$ gives the gate voltage $V_{\mathrm{g}}=V_{\mathrm{c}}$, where a molecular energy level aligns with the equilibrium electrochemical potential of the electrodes. This simple constant interaction model qualitatively describes the measured data of our single-molecule transistor shown in Fig. 5(a). However, it fails to explain the observed decrease of $\mathrm{d} V_{\mathrm{g}} / \mathrm{d} V_{\mathrm{b}}$ with the increase in the peak position at $V_{\mathrm{g}}=0$, if 
$\mathrm{d} V_{\mathrm{g}} / \mathrm{d} V_{\mathrm{b}}<0$, i.e. when both the tip and molecule are negatively charged and the gating of the graphene layer is thus maximized.

To eliminate this deficiency, we include the charging energy of the graphene layer in our second model [Fig. 5(c)]. This model (model 2) is based on the minimization of the total Gibbs free energy of the system. ${ }^{20}$ From the electric field distribution in the transistor geometry, the alignment of the molecular orbitals is determined and the related charge transport properties are derived in a noninteracting single-particle picture, following the Landauer-Büttiker scattering theory. ${ }^{4}$ The details of the calculation are given in ESI S1. $\dagger$

Fig. 6 shows a comparison between $\mathrm{d} I\left(V_{\mathrm{b}}, V_{\mathrm{g}}\right) / \mathrm{d} V_{\mathrm{b}}$ for models 1 and 2. $E_{n}$ and $\varepsilon_{m}$ were chosen to describe the HOMO or LUMO states, and the two separate cases are displayed in each panel. Both models show a similar behavior. For the LUMO states, the peaks in the differential conductance $\mathrm{d} I / \mathrm{d} V_{\mathrm{b}}$ move towards the lower absolute values of $V_{\mathrm{b}}$, if $V_{\mathrm{g}}$ is increased. The opposite is true for the HOMO states. But while $\mathrm{d} V_{\mathrm{g}} / \mathrm{d} V_{\mathrm{b}}$ is constant in model 1 , the charging of the graphene causes a bending of one of the two differential conductance features in model 2. Precisely, these are the peaks at negative $\mathrm{d} V_{\mathrm{g}} / \mathrm{d} V_{\mathrm{b}}$, which result from the alignment of the molecular level with the electrochemical potential of graphene.

(a)

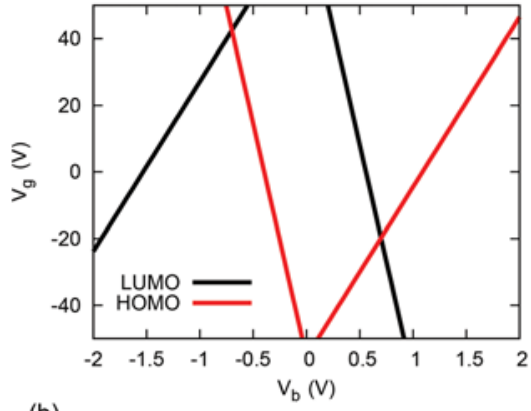

(b)

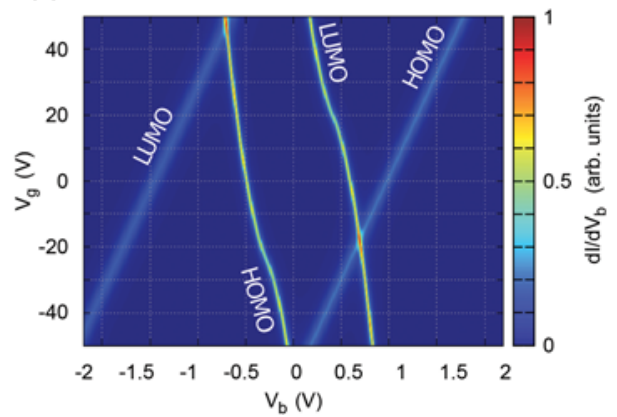

Fig. $6 \mathrm{~d} / / \mathrm{d} V_{\mathrm{b}}$ (in arbitrary units) as a function of $V_{\mathrm{b}}$ and $V_{\mathrm{g}}$ as obtained from (a) model 1 and (b) model 2. Both plots show the shift of peak features related to the molecular HOMO and LUMO states. In model 1, $C_{d}=$ $50 C_{\mathrm{g}}, C_{\mathrm{s}}=140 C_{\mathrm{g}}$ and $E_{n}=0.4 \mathrm{eV}$ (LUMO) and $E_{n}=-0.3 \mathrm{eV}$ (HOMO). In model 2, $\varepsilon_{m}=0.4 \mathrm{eV}$ (LUMO) and $\varepsilon_{m}=-0.3 \mathrm{eV}$ (HOMO), $d_{\mathrm{s}}=1 \mathrm{~nm}, d_{\mathrm{m}}=$ $0.2 \mathrm{~nm}, d_{\mathrm{g}}=300 \mathrm{~nm}, \varepsilon_{1}=4 \varepsilon_{0}, \Phi_{\mathrm{s}}=4.5 \mathrm{eV}, \Phi_{\mathrm{d}}=4.6 \mathrm{eV}, \Phi_{\mathrm{g}}=4.5 \mathrm{eV}, v_{\mathrm{F}}=$ $2 \times 10^{6} \mathrm{~m} \mathrm{~s}^{-1}$ and linewidth broadenings were assumed to be $5 \mathrm{meV}$. The latter have no influence on the movement of $\varepsilon\left(V_{\mathrm{b}} V_{\mathrm{g}}\right)$.
The second model shows a constant slope at $\mathrm{d} V_{\mathrm{g}} / \mathrm{d} V_{\mathrm{b}}>0$, but a variable slope at $\mathrm{d} V_{\mathrm{g}} / \mathrm{d} V_{\mathrm{b}}<0$. In the latter case, the maximum of the slope is located at $0.1 \mathrm{~V}$, which results from the difference in the work functions of the tip $\left(\Phi_{\mathrm{s}}=4.5 \mathrm{eV}\right)$ and graphene $\left(\Phi_{\mathrm{d}}=4.6 \mathrm{eV}\right)$. On both sides of the maximum, $\mathrm{d} V_{\mathrm{g}} / \mathrm{d} V_{\mathrm{b}}$ decreases symmetrically with an increase in the deviations in the peak position. We attribute this to the charge accumulation in the graphene layer induced by the potential applied to the tip, that effectively screens the potential applied to the gate.

\section{Conclusions}

We have demonstrated that a graphene layer on $\mathrm{a} \mathrm{SiO}_{2} / \mathrm{Si}$ substrate can be used to effectively gate the molecular orbitals of CoPc molecules deposited on top of graphene. A careful analysis of the gate voltage induced energy shifts of the molecular features implies that CoPc molecules are electronically decoupled from the graphene substrate. This leads to a situation where coupling of a CoPc molecule to the STM tip is comparable to its coupling to graphene, thus facilitating electronic transport involving only unoccupied molecular states for both tunneling bias polarities. Our experiments provide novel information on the interaction between graphene and organic molecules and are of importance for further studies, which envisage the realization of single molecule transistors with graphene electrodes.

\section{Author contributions}

$\mathrm{RM}, \mathrm{CN}$, and WF fabricated the graphene samples. SB, AG, and CE performed the STM experiments. PE and LG helped in STM characterization. MS and DW performed the theoretical calculations. CS supervised the sample preparation. FB supervised the theoretical calculations. RM, CS, and FB helped in interpreting the STS data. SB and MF conceived and designed the whole study and drafted the manuscript. All authors critically read and approved the manuscript.

\section{Conflicts of interest}

There are no conflicts to declare.

\section{Acknowledgements}

S. B., D. W., F. P., and M. F. acknowledge support through the Collaborative Research Center (SFB 767) "Controlled Nanosystems" of the German Science Foundation (DFG). F. P. acknowledges the Carl-Zeiss foundation for funding. Support by the Swiss NFS, nano-tera.ch, graphene flagship and EUproject HYSENS is greatly acknowledged by R. M., C. N., W. F., and C. S. 


\section{References}

1 N. J. Tao, Nat. Nanotechnol., 2006, 1, 173.

2 M. Ratner, Nat. Nanotechnol., 2013, 8, 378.

3 S. V. Aradhya and L. Venkataraman, Nat. Nanotechnol., 2013, 8, 399.

4 J. C. Cuevas and E. Scheer, Molecular Electronics: An Introduction to Theory and Experiment, World Scientific Publishing Company, 2010.

5 H. Park, A. K. L. Lim, A. P. Alivisatos, J. Park and P. L. McEuen, Appl. Phys. Lett., 1999, 75, 301.

6 M. A. Reed, C. Zhou, C. J. Muller, T. P. Burgin and J. M. Tour, Science, 1997, 278, 252.

7 M. L. Perrin, C. J. O. Verzijl, C. A. Martin, A. J. Shaikh, R. Eelkema, J. H. van Esch, J. M. van Ruitenbeek, J. M. Thijssen, H. S. J. van der Zant and D. Dulić, Nat. Nanotechnol., 2013, 8, 282.

8 H. Park, J. Park, A. K. L. Lim, E. H. Anderson, A. P. Alivisatos and P. L. McEuen, Nature, 2000, 407, 57.

9 J. Park, A. N. Pasupathy, J. I. Goldsmith, C. Chang, Y. Yaish, J. R. Petta, M. Rinkoski, J. P. Sethna, H. D. Abruña, P. L. McEuen and D. C. Ralph, Nature, 2002, 417, 722.

10 W. Liang, M. P. Shores, M. Bockrath, J. R. Long and H. Park, Nature, 2002, 417, 725.

11 A. R. Champagne, A. N. Pasupathy and D. C. Ralph, Nano Lett., 2005, 5, 305.

12 J. E. Grose, E. S. Tam, C. Timm, M. Scheloske, B. Ulgut, J. J. Parks, H. D. Abruña, W. Harneit and D. C. Ralph, Nat. Mater., 2008, 7, 884.

13 A. Aviram, C. Joachim and M. Pomerantz, Chem. Phys. Lett., 1988, 146, 490.

14 C. Joachim, J. K. Gimzewski, R. R. Schlittler and C. Chavy, Phys. Rev. Lett., 1995, 74, 2102.

15 A. Zhao, Q. Li, L. Chen, H. Xiang, W. Wang, S. Pan, B. Wang, X. Xiao, J. Yang, J. G. Hou, et al., Science, 2005, 309, 1542.

16 T. Komeda, H. Isshiki, J. Liu, Y.-F. Zhang, N. Lorente, K. Katoh, B. K. Breedlove and M. Yamashita, Nat. Commun., 2011, 2, 217.
17 M. Ziegler, N. Néel, C. Lazo, P. Ferriani, S. Heinze, J. Kröger and R. Berndt, New J. Phys., 2011, 13, 085011.

18 S. Schmaus, A. Bagrets, Y. Nahas, T. K. Yamada, A. Bork, M. Bowen, E. Beaurepaire, F. Evers and W. Wulfhekel, Nat. Nanotechnol., 2011, 6, 185.

19 S. Kahle, Z. Deng, N. Malinowski, C. Tonnoir, A. FormentAliaga, N. Thontasen, G. Rinke, D. Le, V. Turkowski, T. S. Rahman, et al., Nano Lett., 2012, 12, 518.

20 V. W. Brar, R. Decker, H.-M. Solowan, Y. Wang, L. Maserati, K. T. Chan, H. Lee, C. O. Girit, A. Zettl, S. G. Louie, M. L. Cohen and M. F. Crommie, Nat. Phys., 2011, 7, 43.

21 Y. Wang, V. W. Brar, A. V. Shytov, Q. Wu, W. Regan, H.-Z. Tsai, A. Zettl, L. S. Levitov and M. F. Crommie, Nat. Phys., 2012, 8, 653.

22 A. Riss, S. Wickenburg, L. Z. Tan, H.-Z. Tsai, Y. Kim, J. Lu, A. J. Bradley, M. M. Ugeda, K. L. Meaker, K. Watanabe, T. Taniguchi, A. Zettl, F. R. Fischer, S. G. Louie and M. F. Crommie, ACS Nano, 2014, 8, 5395.

23 M. Ishigami, J. H. Chen, W. G. Cullen, M. S. Fuhrer and E. D. Williams, Nano Lett., 2007, 7, 1643.

24 S. K. Hämäläinen, M. Stepanova, R. Drost, P. Liljeroth, J. Lahtinen and J. Sainio, J. Phys. Chem. C, 2012, 116, 20433.

25 P. Järvinen, S. K. Hämäläinen, K. Banerjee, P. Häkkinen, M. Ijäs, A. Harju and P. Liljeroth, Nano Lett., 2013, 13, 3199.

26 K. W. Hipps, X. Lu, X. D. Wang and U. Mazur, J. Phys. Chem., 1996, 100, 11207.

27 S. Wang, W. Wang, Y. Hong, B. Z. Tang and N. Lin, Phys. Rev. B: Condens. Matter Mater. Phys., 2011, 83, 115431.

28 W. Dou, S. Huang, R. Q. Zhang and C. S. Lee, J. Chem. Phys., 2011, 134, 094705.

29 S. W. Wu, G. V. Nazin, X. Chen, X. H. Qiu and W. Ho, Phys. Rev. Lett., 2004, 93, 236802.

30 B. Warner, F. El Hallak, H. Prüser, J. Sharp, M. Persson, A. J. Fisher and C. F. Hirjibehedin, Nat. Nanotechnol., 2015, 10, 259.

31 J. M. Thijssen and H. S. J. Van der Zant, Phys. Status Solidi $B, 2008,245,1455$. 